\title{
lon heating in an auroral potential structure
}

\author{
A. Anastasiadis ${ }^{1}$, I. A. Daglis ${ }^{1}$, and C. Tsironis ${ }^{2}$ \\ ${ }^{1}$ National Observatory of Athens, Institute for Space Applications and Remote Sensing, 15236 Penteli, Greece \\ e-mail: daglis@space.noa.gr \\ 2 Department of Physics, Aristotle University of Thessaloniki, 54124 Thessaloniki, Greece \\ e-mail: ctsironis@astro.auth.gr
}

Received 15 October 2003 / Accepted 26 February 2004

\begin{abstract}
We investigate the interaction of $\mathrm{O}^{+}$ions with a one-dimensional potential well, using Hamiltonian formulation. Heating of plasma originating in the terrestrial ionosphere plays a catalytic role in solar-driven magnetic storms, which dissipate energy globally within the magnetosphere of the earth. An interesting candidate for ionospheric plasma heating is a potential well located at auroral arcs in the high-latitude magnetosphere. We consider a potential with an exponential form, having a characteristic length $L_{x}$. The oxygen ions drift towards the auroral arc in the presence of a constant magnetic field $B_{z}$ and a constant electric field $E_{y}$. The orbits of individual ions for different initial conditions - phase angle and kinetic energy are traced. Our results show that, depending upon the initial conditions, test particles can be either accelerated or decelerated. Furthermore, we perform a parametric study for the interactions of mono-energetic and Maxwellian type of initial ion distribution - using random phase angle injection of the particles - with respect to our main model parameter, the characteristic length of the potential $L_{x}$. We conclude that for characteristic lengths comparable to twice the ion gyroradius, the $\mathrm{O}^{+}$population is accelerated.
\end{abstract}

Key words. acceleration of particles - plasmas

\section{Introduction}

Plasma heating in planetary magnetospheres is a process of particular astrophysical interest, since plasma systems occur throughout the universe and plasma heating is a key astrophysical process. While boundary conditions and scale sizes vary significantly among different systems, the understanding of plasma heating in one system will contribute fundamentally to studies of other cosmic plasma systems. The sun, our nearest star, drives mass transport, energy dissipation and accelerated particles in the space vicinity of our planet. As a matter of fact, the terrestrial magnetosphere is currently the only region where in-situ measurements of particle acceleration can be made. In the last 4 decades, a plethora of spacecraft measurements have led to a large number of discoveries, and have permitted extensive tests of theory and comparisons of observations with numerical simulations (e.g. Lyon et al. 1981; Baker 1999). In the terrestrial magnetosphere, plasma acceleration is of central importance for the two main complex phenomena, i.e. magnetic storms and magnetospheric substorms, which are closely related in a yet not thoroughly clarified manner (Siscoe 1997).

A large body of literature exists on the transport and acceleration of plasma in the coupled sun-magnetosphereionosphere system (Daglis et al. 1997; Zakharov \& Meister 1997). A particularly interesting case of plasma heating is

Send offprint requests to: A. Anastasiadis, e-mail: anastasi@space.noa.gr the acceleration of relatively cold ionospheric ions (with initial energies of several tens of $\mathrm{eV}$ ) to energies of tens of $\mathrm{keV}$ in relatively short timescales (Rothwell et al. 1992; Daglis \& Axford 1996). Singly-charged oxygen ions, which originate in the ionosphere, have been shown to be dominant during intense magnetic storms (Daglis 1997). Furthermore, statistical studies (e.g., Yau et al. 1984) demonstrated that the auroral ionosphere is the major source of magnetospheric $\mathrm{O}^{+}$ions. Accordingly, there is a pronounced interest in efficient acceleration modes of cold ionospheric plasma in the auroral region.

Upflowing ionospheric ions are interacting with auroral arcs, which are elongated in the east-west direction and often have a Gaussian - U-shape - potential structure (Akasofu 1981; Rothwell et al. 1992). The general case of particle motion in a homogeneous magnetic field $\boldsymbol{B}$ with an orthogonal electric field $\boldsymbol{E}$, which has a gradient $\nabla \boldsymbol{E}$ parallel to $\boldsymbol{E}$, has been studied by Cole (1976). By examining the analytical solutions of the equation of motion, he argued that particle acceleration is possible when the scale length of the potential becomes less than the particle's gyro-radius. Rothwell et al. (1995) examined the behavior of the analytical solutions for a general type of potential. They found that the solutions are very sensitive to the initial conditions and display chaotic behavior on changes of the second derivative of the electric field.

In the present paper, we investigate the particle interactions with a potential structure of an exponential type that was first proposed by Rothwell et al. (1992). In their work, they argued 
that due to the presence of a hyperbolic fixed point in the phase space, some particles are trapped by the potential and some are not, with this effect leading to stochastic ion acceleration. Moreover, they claimed that stochastic heating of a particle population can occur due to the interaction with a potential well of characteristic length comparable to the particle gyrodiameter. In this paper, by using the Hamiltonian formalism, we prove that there is no hyperbolic fixed point that can influence the individual particle trajectories. Furthermore, by performing an extensive study of the interaction of particle distributions with the well, we conclude that the stochastic acceleration of the ions depends upon the initial conditions and the characteristic length of the well.

The organization of this paper is as follows: in Sect. 2, the Hamiltonian of the system is formulated, the equations of motion are derived and a search for equilibrium points is performed. In Sect. 3, our numerical scheme is presented and the results of the simulations are analyzed, while in Sect. 4 our conclusions are summarized.

\section{Hamiltonian formulation}

We assume the presence of an auroral arc potential structure along the $x$-direction with a form

$\varphi=\varphi_{0} \exp \left[-\left(\frac{x}{L_{x}}\right)^{2}\right]$

where $L_{x}$ is the characteristic length of the auroral arc. The corresponding electric field is given by the relation

$E_{x}=-\frac{\mathrm{d} \varphi}{\mathrm{d} x}=\frac{2 \varphi_{0}}{L_{x}^{2}} x \exp \left[-\left(\frac{x}{L_{x}}\right)^{2}\right]$.

Apart from that, we consider that the ions are moving under the presence of a constant background magnetic field $B_{z}$ in the $z$-direction and a constant electric field $E_{y}$ along the $y$-direction. This background electric field causes that the particles will drift in the $x$-direction, towards the auroral arc. The vector potential for these settings is

$\boldsymbol{A}=\frac{1}{2} \boldsymbol{B} \times \boldsymbol{r}=\frac{1}{2}\left(-y B_{z} \boldsymbol{x}+x B_{z} \boldsymbol{y}\right)$.

The motion of an ion with mass $m$ and charge $e$ in the assumed configuration is described by the Lagrangian

$$
\begin{aligned}
L= & \frac{1}{2} m v_{x}^{2}+\frac{1}{2} m v_{y}^{2}-e \varphi_{0} \exp \left[-\left(\frac{x}{L_{x}}\right)^{2}\right]+e y E_{y} \\
& +\frac{e}{2}\left(-y v_{x} B_{z}+x v_{y} B_{z}\right)
\end{aligned}
$$

and the canonical momenta $p_{x}, p_{y}$ are defined as

$$
\begin{aligned}
& p_{x}=\frac{\partial L}{\partial v_{x}}=m v_{x}-\frac{e y B_{z}}{2} \\
& p_{y}=\frac{\partial L}{\partial v_{y}}=m v_{y}+\frac{e x B_{z}}{2}
\end{aligned}
$$

The Hamiltonian function is also calculated from the Lagrangian, by applying a Legendre transformation (Goldstein 1980). The result is

$$
\begin{aligned}
H= & v_{x} p_{x}+v_{y} p_{y}-L=\frac{1}{2 m}\left[\left(p_{x}+\frac{e y B_{z}}{2}\right)^{2}+\left(p_{y}-\frac{e x B_{z}}{2}\right)^{2}\right] \\
& +e \varphi_{0} \exp \left[\left(\frac{x}{L_{x}}\right)^{2}\right]-e y E_{y} .
\end{aligned}
$$

Notice that the Hamiltonian does not depend explicitly on time, and as a consequence it is the energy integral of the motion.

The above formulation provides the canonical equations of the ion motion

$$
\begin{aligned}
& \frac{\mathrm{d} x}{\mathrm{~d} t}=\frac{\partial H}{\partial p_{x}}=\frac{1}{m}\left(p_{x}+\frac{e y B_{z}}{2}\right) \\
& \frac{\mathrm{d} y}{\mathrm{~d} t}=\frac{\partial H}{\partial p_{y}}=\frac{1}{m}\left(p_{y}-\frac{e x B_{z}}{2}\right) \\
& \frac{\mathrm{d} p_{x}}{\mathrm{~d} t}=-\frac{\partial H}{\partial x}=\frac{2 e \varphi_{0}}{L_{x}^{2}} x \exp \left[-\left(\frac{x}{L_{x}}\right)^{2}\right]+\frac{e B_{z}}{2 m}\left(p_{y}-\frac{e x B_{z}}{2}\right) \\
& \frac{\mathrm{d} p_{y}}{\mathrm{~d} t}=-\frac{\partial H}{\partial y}=e E_{y}-\frac{e B_{z}}{2 m}\left(p_{x}+\frac{e y B_{z}}{2}\right)
\end{aligned}
$$

The system of Eqs. (8)-(11) may be written in the form $\dot{X}=$ $f(\boldsymbol{X})$, where $\boldsymbol{X}$ is the column vector of the canonical variables $\left(x, y, p_{x}, p_{y}\right)$ and $f(\boldsymbol{X})$ is the vector field

$$
f(\boldsymbol{X})=\left[\begin{array}{c}
\frac{1}{m}\left(p_{x}+\frac{e y B_{z}}{2}\right) \\
\frac{1}{m}\left(p_{y}-\frac{e x B_{z}}{2}\right) \\
\frac{2 e \varphi_{0}}{L_{x}^{2}} x \exp \left[-\left(\frac{x}{L_{x}}\right)^{2}\right]+\frac{e B_{z}}{2 m}\left(p_{y}-\frac{e x B_{z}}{2}\right) \\
e E_{y}-\frac{e B_{z}}{2 m}\left(p_{x}+\frac{e y B_{z}}{2}\right)
\end{array}\right] .
$$

This form of the motion equations is very helpful in determining the system equilibria. If an equilibrium point $\boldsymbol{X}_{\mathrm{ec}}$ exists, it must satisfy the familiar condition $f\left(\boldsymbol{X}_{\mathrm{ec}}\right)=0$ (i.e. Lichtenberg $\&$ Lieberman 1992). This translates to the following relations for the $\boldsymbol{X}_{\mathrm{ec}}$ components

$$
\begin{aligned}
& \frac{1}{m}\left(p_{x \mathrm{ec}}+\frac{e y_{\mathrm{ec}} B_{z}}{2}\right)=0 \\
& \frac{1}{m}\left(p_{y \mathrm{ec}}-\frac{e x_{\mathrm{ec}} B_{z}}{2}\right)=0 \\
& \frac{2 e \varphi_{0}}{L_{x}^{2}} x_{\mathrm{ec}} \exp \left[-\left(\frac{x_{\mathrm{ec}}}{L_{x}}\right)^{2}\right]+\frac{e B_{z}}{2 m}\left(p_{y \mathrm{ec}}-\frac{e x_{\mathrm{ec}} B_{z}}{2}\right)=0 \\
& e E_{y}-\frac{e B_{z}}{2 m}\left(p_{x \mathrm{ec}}+\frac{e y_{\mathrm{ec}} B_{z}}{2}\right)=0
\end{aligned}
$$

By inserting Eq. (13) into Eq. (16) we obtain $e E_{y}=0$, which is a relation that cannot be valid. Consequently, no equilibrium points exist in the phase space of the system, because of the electric field that causes the particle drift. This result is in conflict with the conclusion of Rothwell et al. (1992) that a hyperbolic fixed point exists near the boundaries of the potential well. The only argument used to support the existence of the 
fixed point, namely the presence of the separatrices, in the particle motion was based on the form of the phase-space projections. These projections do not reveal the true picture of the phase space. In addition, not even the use of Poincare surfaces of section can be useful, as the ion motions cannot be followed in a standard way because they have mixed transient features.

\section{Results}

In this section we present our numerical scheme and our results concerning the heating of the ions. We numerically integrated the equations of motion (8)-(11) using a 4th order Runge-Kutta algorithm with step $h=0.01$ (Press et al. 1986). The integration time for each orbit was not standard. We are going to use the term upstream to specify the region in front of the potential well (i.e. $x<0$ ) and the term downstream for the region behind the well (i.e. $x>0$ ). The ions were followed towards the potential well and collected at a far distance $10 L_{x}$ downstream of the well. The calculations were performed for a certain choice of system parameters (see Rothwell et al. 1992): the constant electric field was chosen $E_{y}=1 \mathrm{mV} / \mathrm{m}$, while the constant magnetic field was $B_{z}=144 \mathrm{nT}$ and the auroral arc potential minimum was taken $\phi_{0}=-3 \mathrm{kV}$. The characteristic length $L_{x}$ was used as a parameter and varied in the range [10-200] km.

The ion energy,

$H=E=\frac{1}{2} m\left(v_{x}^{2}+v_{y}^{2}\right)+e \phi(x)-e y E_{y}$

is constant during each step of the integration. For an accuracy criterion of our numerical simulations, we followed the maximum error in calculating the total energy. In all integrations, it was found to be less than $10^{-3}$ of the initial energy.

In Fig. 1 we present the orbits of three oxygen ions $\mathrm{O}^{+}$towards an auroral arc of characteristic length $L_{x}=50 \mathrm{~km}$. The ions are injected at the point $(x, y)=\left(-10 L_{x}, 0\right)$ upstream and have the same initial energy $E_{0}=200 \mathrm{eV}$, but different phase angles (a) $\theta=20^{\circ}$, (b) $\theta=60^{\circ}$ and (c) $\theta=80^{\circ}$. We use the term "phase angle" to refer to the angle formed by the initial velocity components in the $x, y$ plane, given by

$\theta=\arctan \left(\frac{v_{y 0}}{v_{x 0}}\right)$.

This relation is used for calculating the initial conditions $v_{y 0}, v_{x 0}$ from the given values for $E_{0}, \theta$. Note that the spatial coordinates are normalized to the characteristic length $L_{x}$.

From these figures, it is clear that ions are trapped by the potential for a part of their orbit. The duration of the trapping depends sensitively upon the initial conditions, namely the phase angle. The interaction with the auroral arc causes a displacement in the $y$-direction, either positive or negative. This displacement is equivalent to a kinetic energy gain or loss, because the total energy is constant and so any dynamical energy variation is reflected in the kinetic energy. This is certified by the following relation

$\Delta E_{\mathrm{k}}=e E_{y} \Delta y$

which is a consequence of the energy conservation law, applied at points far upstream and downstream of the auroral arc. Thus,
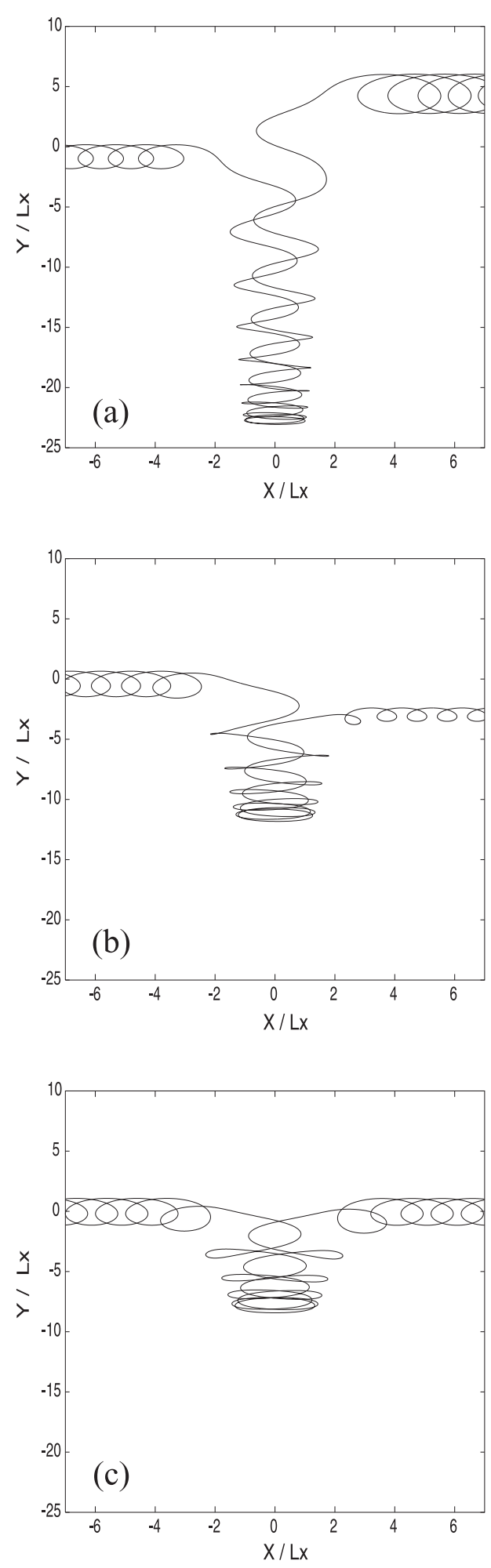

Fig. 1. Ion orbit in the $(x, y)$ plane for initial energy $E_{0}=200 \mathrm{eV}$ and phase angles a) $\theta=20^{\circ}$ b) $\theta=60^{\circ}$ c) $\theta=80^{\circ}$. The coordinates $x, y$ are normalized to the characteristic length $L_{x}$.

the ion with phase angle $20^{\circ}$ is accelerated and the one with $60^{\circ}$ is decelerated, while the ion injected with $\theta=80^{\circ}$ does not show a significant energy variation.

The trapping of ions can also be deduced by visualizing the phase-space projections $\left(x, p_{x}\right)$ and $\left(y, p_{y}\right)$ of the ion evolution. 

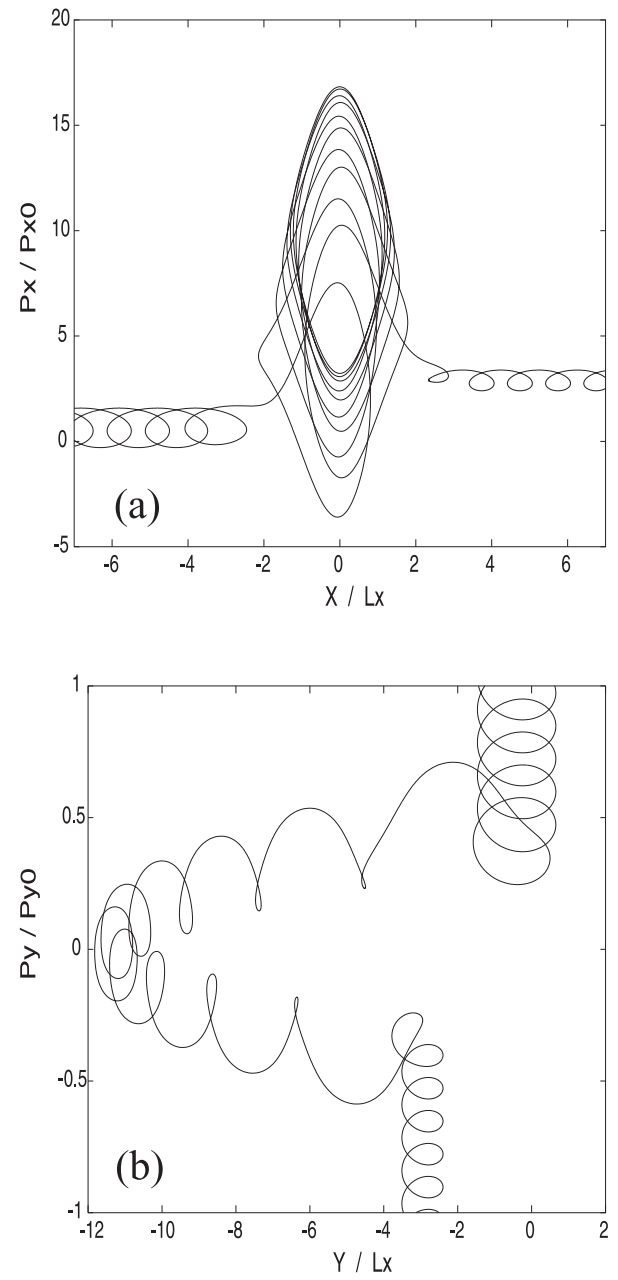

Fig. 2. Phase space projections a) $\left(x, p_{x}\right)$ and b) $\left(y, p_{y}\right)$ of the ion motion for initial energy $E_{0}=200 \mathrm{eV}$ and phase angle $\theta=60^{\circ}$. The coordinates $x, y$ are normalized to the characteristic length $L_{x}$ and the canonical momenta $p_{x}, p_{y}$ to their initial values $p_{x 0}, p_{y 0}$.

Such projections of course do not retain all the properties of the real phase space, but they can give a general view of the ion kinetics. These are given in Fig. 2 for (a) $\left(x, p_{x}\right)$ and (b) $\left(y, p_{y}\right)$ for an ion injected with phase angle $\theta=60^{\circ}$ with the rest of the parameters being the same as above. The coordinates $x, y$ are normalized to the characteristic length $L_{x}$, while the canonical momenta $p_{x}, p_{y}$ are normalized to their initial values $p_{x 0}, p_{y 0}$. The trapping of the ions in the region of interaction with the potential is clearly seen in both plots.

In order to perform a more realistic investigation of the acceleration mechanism we consider the interaction of ion population with the potential. First we investigate the dependence of the ion acceleration upon the initial phase angle, by performing the following simulation. We trace the orbits of a mono-energetic distribution of $N=10000$ ions, with initial energy of $E_{0}=200 \mathrm{eV}$, injected with phase angles uniformly distributed in $(-\pi / 2, \pi / 2)$, corresponding to velocity vectors pointing towards the well and starting at a given space point (i.e. $\left.(x, y)=\left(-10 L_{x}, 0\right)\right)$ upstream of the well. In Fig. 3, the exit kinetic energy, normalized to initial energy, is plotted as a function of the phase angle for $L_{x}=100 \mathrm{~km}$.

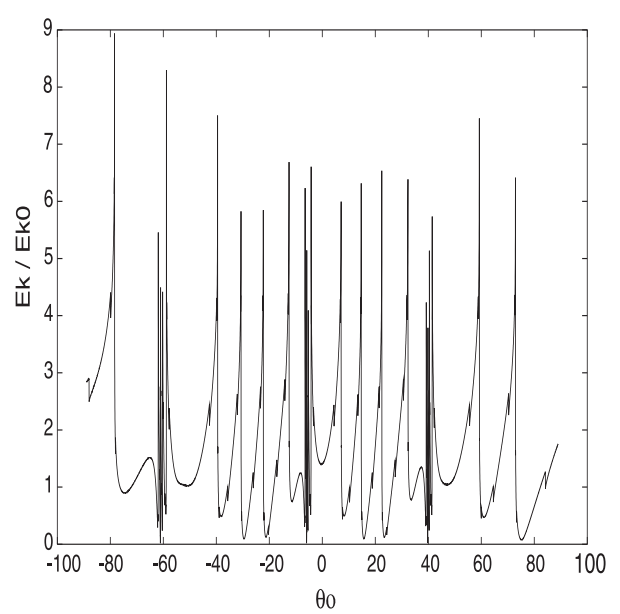

Fig. 3. Normalized ion exit kinetic energy as a function of the phase angle $\theta$ for characteristic length $L_{x}=100 \mathrm{~km}$. The initial energy of the ion is $E_{0}=200 \mathrm{eV}$, while $\theta$ is uniformly distributed in $(-\pi / 2, \pi / 2)$.

From this figure, it becomes apparent that the ion acceleration does not have a monotonic dependence on $\theta$. The exit energy varies very rapidly in the region of values $(-\pi / 2, \pi / 2)$. For some angles the exit energy is very large, e.g. for $-60^{\circ}$ or $40^{\circ}$, but for their neighboring values the energy is much smaller. Thus, large intervals of preferable phase angles, where acceleration is more intense, do not exist. For checking this further, a Fourier transform was performed on the data; as a result, no dominant phase angles were found to exist.

Furthermore, we trace mono-energetic distributions of ions, with initial kinetic energy $E_{0}=200 \mathrm{eV}$ and random phase angles, for several values of the characteristic length $L_{x}$. In Fig. 4 the kinetic energy variation - the ratio of the final to the initial kinetic energy - is given as a function of $L_{x}$. The plot is formed as follows: at each $L_{x}$ value, a mono-energetic ensemble of $N=1000$ ions is injected at the same point in space upstream of the well, with random phase angles, and it is collected at $x=10 L_{x}$ downstream. The vertical bars of dots in the plot correspond to the kinetic energy of individual particles, while the solid line stands for the ensemble mean of the kinetic energy.

For characteristic lengths in the range 80 to $130 \mathrm{~km}$, a large fraction - up to $70 \%$ - of the injected particles is accelerated, reaching energies even up to 6 or 7 times the initial energy. This is reflected also in the mean normalized energy of the ensemble, which takes values fairly larger than 1 , within $[1.5,1.8]$. These values indicate that the majority of the ensemble is accelerated. Notice that such $L_{x}$ values are approximately equal to twice the ion gyroradius (i.e. $L_{x} \sim D_{\mathrm{gyr}}=2 R_{\mathrm{gyr}} \simeq 114 \mathrm{~km}$ ), where the ion gyroradius is given from the general relation

$R_{\mathrm{gyr}}=\frac{m v_{0}}{e B_{z}}=\frac{\left(2 m E_{k 0}\right)^{1 / 2}}{e B_{z}}$.

Our first conclusion is that acceleration is more intense when the scale of the ion gyro-diameter is comparable to the characteristic length of the auroral arc. On the contrary, in cases where these scales are not comparable, only a small fraction of the ensemble - around $45 \%$ - is accelerated. This is due to the character of the ion-arc interaction for these cases: when $L_{x}$ is 


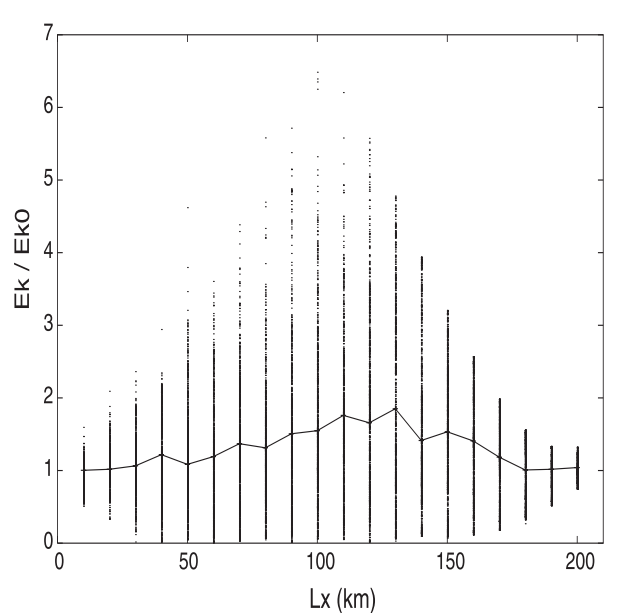

Fig. 4. Kinetic energy variation $E_{\mathrm{kf}} / E_{\mathrm{k} 0}$ as a function of $L_{x}$ (in $\mathrm{km}$ ). Each $L_{x}$-bar contains the ratios of $N=1000$ particles injected with initial energy $E_{0}=200 \mathrm{eV}$ and phase angles in $(-\pi / 2, \pi / 2)$. The solid line corresponds to the ensemble mean variation $\left\langle E_{\mathrm{kf}} / E_{\mathrm{k} 0}\right\rangle$.

small compared to the scale of the ion gyro-diameter, the ions are perturbed rather than trapped by the potential. On the other hand, in cases where $L_{x} \gg D_{\mathrm{gyr}}$, the motion is very close to following the equipotential lines of the arc structure due to the auroral arc being relatively wide. Apparently, from our study, it is not certain if there will be a significant net energy gain for the ions. This depends heavily on the initial conditions of the ensemble, as well as on the size of the auroral arc.

In order to visualize the above in more detail, we focus on some indicative values of $L_{x}$ and form the exit kinetic energy distribution. In Fig. 5 we present the distribution function of the final kinetic energy for similar mono-energetic ensembles of $N=10000$ ions. This is done for three characteristic length cases: (a) $L_{x}=10 \mathrm{~km}$, (b) $L_{x}=100 \mathrm{~km}$ and (c) $L_{x}=190 \mathrm{~km}$. Notice that these figures are not similarly scaled because of the different magnitude of the distribution function for each case.

For $L_{x}=100 \mathrm{~km}$, which is of the order of the ion gyrodiameter, acceleration is intense. This is seen in Fig. 5b, where a large part of the ensemble gains energy more than twice its initial value. Figures 5a,c verify that for well lengths not comparable to the gyroradius, acceleration is of smaller magnitude. Notice in these two cases that the exit kinetic energies reside in a small region of values around 1 .

Next, we examine what happens to a group of ions injected with a Maxwellian kinetic energy distribution upstream of the potential well (solid curve in Fig. 6). Figure 6 shows the kinetic energy distribution, of $N=10000 \mathrm{O}^{+}$ions which are injected with initial thermal energy $E_{\mathrm{th} 0}=200 \mathrm{eV}$ and random phase angles, for the three cases (a) $L_{x}=10 \mathrm{~km}$ (b) $L_{x}=100$ (c) $L_{x}=$ $190 \mathrm{~km}$. All other system parameters remain the same.

The results resemble to the ones found during the study of the mono-energetic ensemble interactions. In cases (a) and (c), corresponding to characteristic lengths $L_{x}$ clearly smaller or greater than $D_{\text {gyr }}$, no significant heating is observed. The initial and final distributions are very close; especially for the high-energy part of the initial distribution - values above 4 - we notice that it appears almost the same as before. This happens because ions with high energies do not get affected
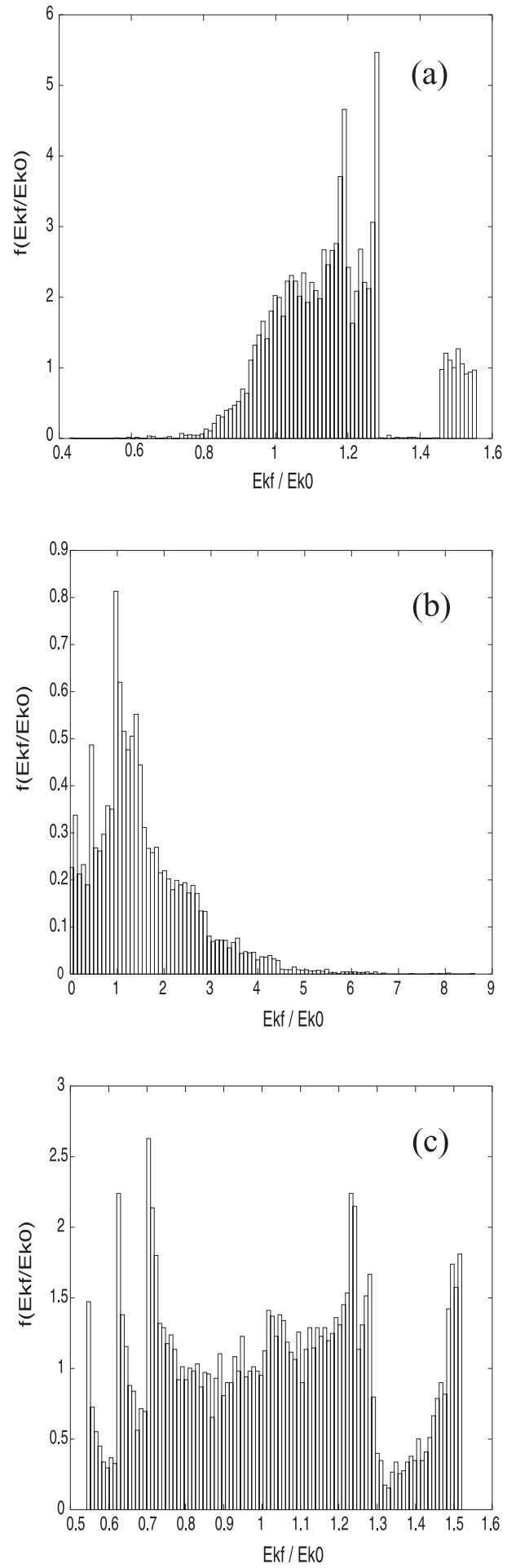

Fig. 5. Kinetic energy distribution of $N=10000$ ions of initial energy $E_{0}=200 \mathrm{eV}$ for a) $L_{x}=10 \mathrm{~km}$, b) $L_{x}=100 \mathrm{~km}$ c) $L_{x}=190 \mathrm{~km}$. The kinetic energy values are normalized to $E_{0}$.

considerably by the potential, as their gyro-radii are too big or too small compared to the characteristic length $L_{x}$. On the contrary, for $L_{x}=100 \mathrm{~km}$ significant heating occurs, at least as seen from Fig. 6b. The final distribution is approximately Maxwellian but it is shifted to high energies. This is an indication of a shift in the thermal equilibrium of the system. 

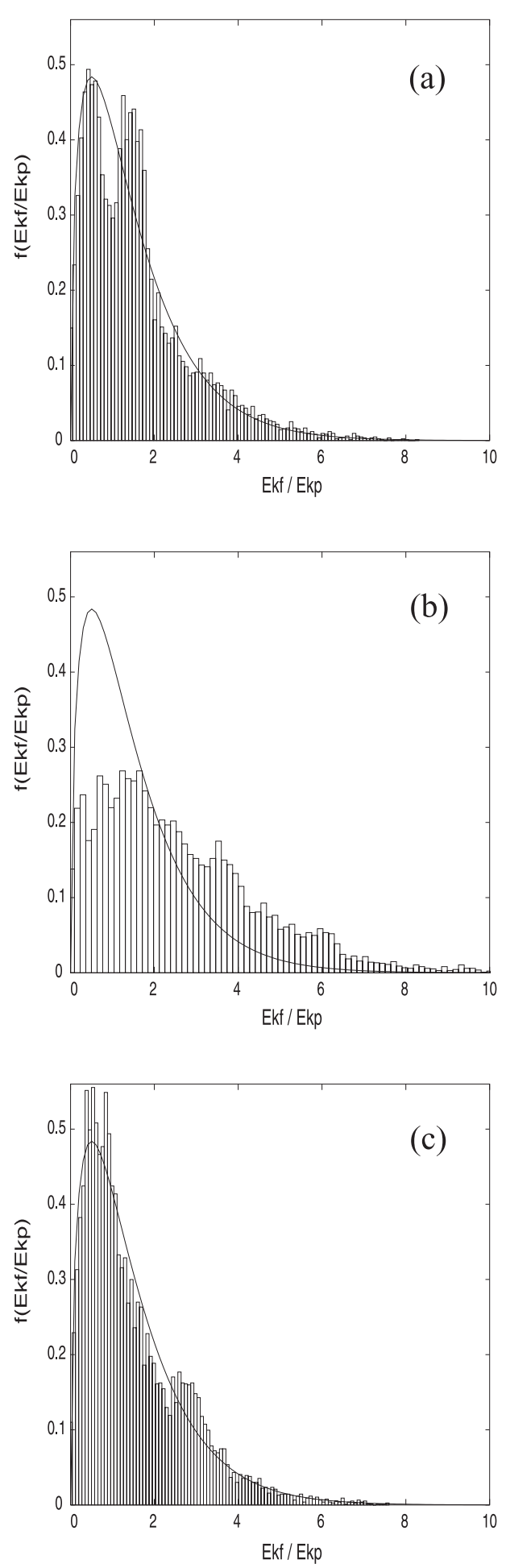

Fig. 6. Distribution of $N=10000$ ions of initial thermal energy $E_{\mathrm{th} 0}=$ $200 \mathrm{eV}$ for a) $\left.L_{x}=10 \mathrm{~b}\right) L_{x}=100$ c) $L_{x}=190 \mathrm{~km}$. The initial distribution is the solid curve. The energies are normalized to $2 E_{\mathrm{th} 0} / 3$.

In order to certify these aspects quantitatively, we fit with the probability density function of the Maxwell distribution,

$$
f(E)=\left(\frac{6 E}{\pi E_{\mathrm{th}}}\right)^{1 / 2} \exp \left(-\frac{3 E}{2 E_{\mathrm{th}}}\right)
$$

with the only parameter the thermal energy. The result for $L_{x}=$ $10 \mathrm{~km}$ was $E_{\mathrm{th}}=216.2 \mathrm{eV}$, or 1.08 in terms of the ratio of

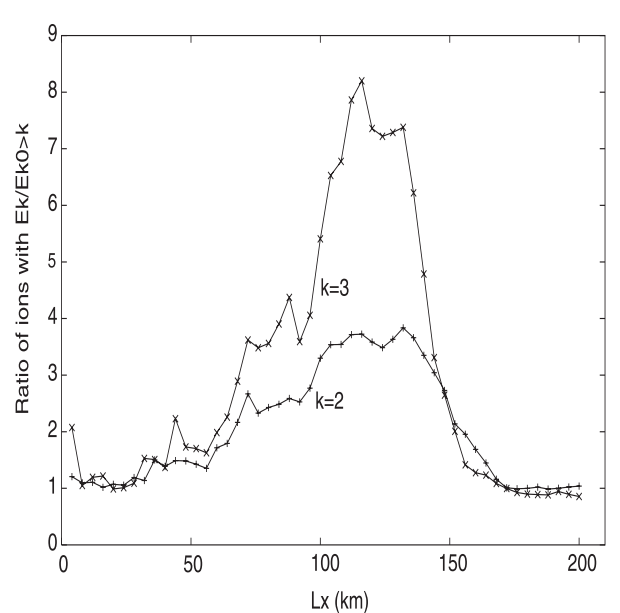

Fig. 7. Ratio of - initially Maxwell-distributed - ions with exit kinetic energies larger than $k E_{\text {tho }}$ vs. ions with initial kinetic energies larger than $k \cdot E_{\text {th0 }}$ as a function of $L_{x}$ for $k=2$ (I) and $k=3$ (II). The ions used are $N=10000$ of thermal energy $E_{\text {th } 0}=200 \mathrm{eV}$ with random initial phases. The energy values are normalized to $2 E_{\text {tho }} / 3$.

the new to the old thermal energy, while for $L_{x}=190 \mathrm{~km}$ it was $E_{\text {th }}=199.8 \mathrm{eV}$ (a ratio 0.9). These values for the final thermal energy correspond to insignificant heating. For the case $L_{x}=100 \mathrm{~km}$, the fitting gave for the thermal energy a result $E_{\mathrm{th}}=375.5 \mathrm{eV}$, or 1.87 for the ratio of the new and old thermal energies, which corresponds to heating of the ensemble. This value is in agreement with the mean energy variation calculated in forming Fig. 4.

Considering these facts, we could say that for $L_{x} \sim D_{\text {gyr }}$ the ion population is heated during its passage through the well. This heating is the result of the stochastic acceleration that affects a part of the ion ensemble due to its interaction with the auroral arc structure. In order to understand the ion heating in more detail, we extended our study of the potential interaction with Maxwell distributions by using a scheme analogous to the one presented in Fig. 4. We calculate the ratio of ions with exit kinetic energies $E_{\mathrm{kf}}$ larger than $k$ times the ensemble thermal energy $E_{\mathrm{th} 0}$ versus ions with initial kinetic energies larger than $k \cdot E_{\text {th0 } 0}$ for various values of $L_{x}$. In Fig. 7 the results are presented for two cases of $k, k=2$ and $k=3$. A number of $N=10000$ test particles are used. Their thermal energy is $E_{\mathrm{th} 0}=200 \mathrm{eV}$ and they are injected with uniformly random initial phases. The kinetic energy values are normalized to $2 E_{\mathrm{th} 0} / 3$.

In both cases, the calculated ratio is maximum in the area of characteristic lengths [100-130] km, comparable to $D_{\text {gyr }}$. That was expected according to the analysis made above, both for mono-energetic as well as for Maxwellian ion injections. The ratios of the $k=3$ case are larger than the ones for $k=2$, even reaching up to values near 8 . That happens because the particles having initial energies larger than $3 E_{\text {th0 }}$ are less than the ones with $E_{\mathrm{k} 0}>2 E_{\mathrm{th} 0}$, while the respective numbers for the final kinetic energies are comparable. Consequently, the ratios of the case $k=3$ have smaller denominators than the ones of $k=2$, while the numerators are of similar magnitude, and so the former ratios take larger values. 


\section{Summary and conclusions}

In this paper, the nonlinear interaction of oxygen ions with an auroral arc has been studied, using the Hamiltonian formulation. The potential was of an exponential type with characteristic length $L_{x}$. In this system, the ions are drifting due to two constant background fields, a magnetic $B_{z}$ and an electric $E_{y}$. The equations of motion were derived by the means of the Hamiltonian function. The Hamiltonian is a constant of motion - the energy integral - as no explicit time-dependence exists.

A search for phase-space equilibrium points was performed; the conclusion was that no such points exist, due to the existence of the drift-causing electric field $E_{y}$. This result is in conflict with the work of Rothwell et al. (1992), where it was claimed that a hyperbolic fixed point exists near the boundaries of the potential well. The arguments used to support the existence of the fixed point were mainly based on the form of phase-space projections. For systems with more than one dimension, as in our case, projections do not reveal the true nature of the system phase space.

The orbits of individual ions for different initial conditions - phase angle and kinetic energy - were traced. It was found that, depending upon the initial conditions, the ions are trapped by the potential well for small or large parts of their motion. After the interaction with the auroral arc, the ions appear displaced in the $y$-direction. This displacement is equivalent to a kinetic energy variation, because the total energy is constant. Based on these results, we can conclude that the acceleration process of ions exhibits a stochastic nature, due to its strong dependence upon the initial conditions.

Furthermore, we performed a parametric study for the interactions of mono-energetic and Maxwellian type of ion distributions with respect to our model parameter $L_{x}$, using random phase angle injection of the particles. The final distributions of the mono-energetic ensembles show that, for $L_{x}$ values comparable to the ion gyro-diameter, the ion acceleration is intense. A large fraction of the ensemble reaches final values more than 2 times its initial energy. This is seen also in the mean normalized energy of the particles, which is fairly larger than 1 . When the scales $L_{x}, D_{\mathrm{gyr}}$ are not comparable, only a small fraction of the ions is accelerated. This is due to the nature of the interaction for these cases: when $L_{x} \ll D_{\text {gyr }}$, the ions are trapped by the potential for less that a gyro-period. Reversely, when $L_{x} \gg D_{\mathrm{gyr}}$, the ions follow the equipotential lines of the arc structure.

The possibility of the heating of the ions was examined by following ions injected with Maxwellian kinetic energy distributions. Significant heating was observed only for $L_{x}$ comparable to the ion gyro-diameter. In the cases where the characteristic length is not comparable to $D_{\mathrm{gyr}}$, no significant heating was found. This behavior was verified quantitatively, by estimating the distribution function after the interaction.

Considering these facts, we conclude that for $L_{x} \sim D_{\mathrm{gyr}}$ the ion population is accelerated during its interaction with the potential well. This acceleration is of a stochastic nature, depending upon the initial conditions of the injected distribution and leads to heating of the particle population.
Our results contribute to the understanding of the changing flow of energy and matter from the Sun to planetary environments and of its effects. In particular, they contribute to the understanding of the response of Earths magnetosphere to solar drivers, as they relate to the chain of action/reaction processes that regulate solar energy transfer into and through the magnetosphere-ionosphere system. As already mentioned, ionospheric-origin $\mathrm{O}^{+}$ions dominate the inner magnetosphere during intense magnetic storms. Mass loading of the plasma sheet due to the high abundance of $\mathrm{O}^{+}$ions can regulate instability growth and the unloading of energy in the geospace environment, especially in the case of substorm series.

Since the ionospheric ions are initially rather cold, the question of efficient acceleration of the ionospheric ions and associated extraction into the magnetosphere is of central interest. Presumably, a variety of successive acceleration mechanisms act on the ionospheric ions to raise the particle energy from about an $\mathrm{eV}$ to tens of $\mathrm{keV}$ (Daglis \& Axford 1996). To comprehensively understand the solar wind-magnetosphereionosphere coupling, we have to investigate the acceleration of ionospheric ions in full detail. We know that electric fields in the magnetosphere depend on the solar wind-magnetosphere coupling through the dayside magnetic field merging and the subsequent magnetospheric convection on the nightside. The next step in our work will be to relate high-latitude electric fields and the associated ion acceleration to solar and interplanetary magnetic field parameters, in order to find the most effective solar and solar wind drivers for delivering ionospheric plasma to the inner magnetosphere.

Acknowledgements. We would like to thank Dr. H. Isliker for many stimulating discussions on particle dynamics and Dr. J. McFadden for helpful comments. This work was partially supported by the Association Euratom-Hellenic Republic.

\section{References}

Akasofu, S.-I. 1981, Geophys. Monogr. Ser., 25, 1

Baker, D. N. 1999, Phys. Plasmas, 6 (5), 1700

Cole, K. D. 1976, Planet. Space Sci., 24, 515

Daglis, I. A. 1997, Geophys. Monogr. Ser., 98, 107

Daglis, I. A., \& Axford, W. I. 1996, J. Geophys. Res., 101, 5047

Daglis, I. A., Axford, W. I., Sarris, E. T., Livi, S., \& Wilken, B. 1997, Sol. Phys., 172, 287

Goldstein, H. 1980, Classical Mechanics, 2nd ed. (New York: Addison-Wesley)

Lyon, J. G., Brecht, S. H., Huba, J. D., Fedder, J. A., \& Palmadesso, P. J. 1981, Phys. Rev. Lett., 46, 1038

Lichtenberg, A. J., \& Lieberman, M. A. 1992, Regular and Chaotic Dynamics, 2nd ed. (New York: Springer-Verlag)

Press, W. H., Flannery, B. P., Teukolsky, S. A., \& Vettering, W. T. 1986, Numerical Recipes in Fortran, 4th ed. (New York: Cambridge University Press)

Rothwell, P. L., Silevitch, M. B., Block, L. P., \& Fälthammar, C.-G. 1992, J. Geophys. Res., 97, 19333

Rothwell, P. L., Silevitch, M. B., Block, L. P., \& Fälthammar, C.-G. 1995, J. Geophys. Res., 100, 14785

Siscoe, G. 1997, Nature, 390, 448

Yau, A. W., Whalen, B. A., Peterson, W. K., \& Shelley, E. G. 1984, J. Geophys. Res., 89, 5507

Zakharov, V. E., \& Meister, C. V. 1997, Astron. Nachr., 318, 51 